\title{
Prediction of Kick Direction from Kinematics during the Soccer Penalty Kick
}

\author{
Yumeng Li (Corresponding author) \\ Department of Kinesiology, University of Georgia, Athens, Georgia, USA \\ 330 River Rd, Athens, GA30605, USA \\ E-mail: yumengli@uga.edu \\ Marion J.L. Alexander \\ Faculty of Kinesiology and Recreation Management, University of Manitoba, Winnipeg, Canada \\ 306 Max Bell Center, Winnipeg, R3T 2N2, Canada \\ E-mail: marion.alexander@umanitoba.ca \\ Cheryl M. Glazebrook \\ Faculty of Kinesiology and Recreation Management, University of Manitoba, Winnipeg, Canada \\ 319 Max Bell Center, Winnipeg, R3T 2N2, Canada \\ E-mail: cheryl.glazebrook@umanitoba.ca \\ Jeff Leiter \\ Pan Am Clinic, Winnipeg, Canada \\ 75 Poseidon Bay, Winnipeg, R3M 3E4, Canada \\ E-mail: jleiter@panamclinic.com
}

Received: 01-08- 2015

doi:10.7575/aiac.ijkss.v.3n.4p.1
Accepted: 18-09- 2015

Published: 31-10- 2015

\begin{abstract}
Background: Speed and direction of the ball are key factors in successful soccer penalty kicks. The kinematics that contribute to the kick direction are unclear. Purpose: The purpose of the study was (1) to compare the differences in instep kick kinematics between left and right kick directions; (2) to determine the significant factors that predict kick direction. Method: Four digital video cameras $(80 \mathrm{~Hz})$ collected data from eleven experienced female soccer players during instep penalty kicks to the left and right. Video analysis software (Dartfish Team Pro 6.0) was used to process and analyze the video files. Results: Paired t-tests indicated that several variables before ball contact were different. The linear regression included three variables (support foot orientation, support foot position behind the ball and approach angle) to best predict kick directions $\left(\mathrm{R}^{2}=75.6 \%, \mathrm{p}<.01\right)$. Conclusion: The results may be useful for goalkeepers to anticipate kick direction before ball contact to gain a better chance to save the penalty kick.
\end{abstract}

Keywords: Instep kick, Anticipation, Goalkeeping

\section{Introduction}

For a penalty kick in soccer the player kicks a stationary ball located $11 \mathrm{~m}$ away from the goal line. Two types of kicks are commonly used during penalty kicks: side-foot kick and instep kick (Nunome, Asai, Ikegami, \& Sakurai, 2002). The side-foot kick is frequently used to gain accuracy, whereas instep kick is used to increase ball speed (Nunome et al., 2002). The ball speed can be up to $30 \mathrm{~m} / \mathrm{s}$ (Andersen \& Dörge, 2011), resulting in only $0.3-0.4$ seconds from ball contact to when the ball passes the goal line. The level of difficulty in penalty defense was highlighted by Bar-eli, Azar, \& Ritov, (2007) who analyzed 286 penalty kicks in top leagues and worldwide championships. They reported that $80 \%$ of the penalty kicks resulted in a goal being scored (Bar-eli, Azar, \& Ritov, 2007).

In order to increase the chance to save penalty kicks for goalkeepers, several strategies were proposed by previous authors. Kuhn (1987) described late strategy and early strategy. The late strategy indicated that goalkeepers move to one side or another at the moment of ball contact or immediately afterwards, whereas the early strategy involved moving before ball contact (Kuhn, 1987). The late strategy was suggested as a better strategy with a higher rate of successful saves (Kuhn, 1987; Morya, Bigatao, Lees, \& Ranvaud, 2005). Another specific strategy was proposed by Bar-Eli et al. (2007). They suggested the optimal strategy for goalkeepers may be to stay in the center of the goal. The probability of saving the penalty kick is higher when the goalkeeper remains in the center $(33.3 \%)$ compared to jumping to the left (14.2\%) or right (12.6\%) (Bar-eli et al., 2007).

Goalkeepers should also strive to anticipate ball direction using visual cues before ball contact (Lees \& Owens, 2011). Savelsbergh and colleagues (2002) investigated differences in anticipation and visual search behavior between expert 
and novice goalkeepers during the penalty kick. They found that experts were more accurate in predicting the direction of the penalty kick and waited longer before initiating a response (Savelsbergh, Williams, Van Der Kamp, \& Ward, 2002). Savelsbergh et al. (2002) suggested the ball areas (including support leg, kicking leg and ball) were more informative compared to arms, trunk and pelvis area, particularly as the moment of ball contact approached. This finding was also supported by Dicks and colleagues (2011) in which they suggested goalkeepers would benefit from learning to ignore early information (e.g. approach) and use later information that is just before the initiation of the kicking action.

On the other hand, high ball-speed and accurate cunning direction (e.g. corner of the goal) would give little chance for goalkeepers to save penalty kicks. There are various studies focused on factors that can contribute to ball speed (Dörge, Andersen, Sørensen, \& Simonsen, 2002; Katis \& Kellis, 2010; Nunome et al., 2002). However, there are few studies investigating kick directions in penalty kicks (Scurr \& Hall, 2009; van der Kamp, 2006). Most of these studies focused on the kick accuracy. To our knowledge, there is only one study that quantitatively described the postural cues in kicking that may be used by goalkeepers to save penalty kicks (Lees \& Owens, 2011). These authors compared the movement of three different kicks: low side-foot kick to the left corner (relative to the kicker), low side-foot kick straight ahead and a low instep kick straight ahead. They suggested that the support foot orientation on the ground is the best cue to predict kick direction (Lees \& Owens, 2011). However, due to the limits of using a laboratory space, the researchers did not study kicks to the right. Players tend to kick the ball to the side of the goal and almost $40 \%$ penalty kicks are to the right (Bar-eli et al., 2007). To our knowledge, there has been no study comparing the differences in the instep kick movements involved in altering kick directions, so the key factors that contribute to kick direction are still unclear. Therefore, the purpose of the present study was (1) to compare the differences in instep kick kinematics between two kick directions: left and right; (2) to determine the significant kinematic factors that predict kick direction. Understanding of the relationship of these factors may be useful in preparing goalkeepers to anticipate kick direction before ball contact, giving them increased time in which to react and stop the ball. We hypothesize that kicking leg, support foot and pelvis kinematics would display differences between different kick directions and those kinematic variables could be used to predict the kick direction.

\section{Methods}

\subsection{Participants}

Eleven female participants (height $166 \pm 8 \mathrm{~cm}$, weight $61 \pm 7 \mathrm{~kg}$, age $21 \pm 1 \mathrm{yr}$ ) were recruited from the university soccer team. All participants were right foot dominant players without any injuries in the six months prior to the study. All participants were skilled with an average of $13.4 \pm 1.9$ years of experience in soccer training. Consent forms were signed by the participants, following the university ethics protocol. The participants wore their own outdoor soccer cleats to perform the instep kicks.

\subsection{Instrumentation}

Four digital video cameras $(80 \mathrm{~Hz}, 640 \times 480$ pixels, shutter speed $=1 / 500 \mathrm{~s}$, Fijifilm EXR $)$ were used to film the kick movements (Figure 1). One camera was set up five meters on the right side the ball and perpendicular to the sagittal plane of the kick movement. Because the participants were all right-foot dominant, the camera was on the right side to capture the movement that occurred in the sagittal plane. Another camera was set up behind the net of the goal, which captured the participants' movement that occurred in the frontal plane. The third camera was suspended 3.5 meters above the ground and directly above the ball. This camera was mounted on a lightweight tripod that was securely fixed on the end of a steel pipe. The other end of the pipe was anchored on the ground. This overhead view camera captured the movement that occurred in the transverse plane. The fourth camera was behind the ball. This camera captured the pathway of the ball to the goal in order to determine successful trials to analyze. This camera setup and arrangement ensured the relatively good quality of each frame in the video, so that all movements of interest could be viewed and analyzed. A ' $T$ ' shape tape marker was placed on the ground close to the penalty spot to define right (+)/left (-) $\mathrm{x}$-axis and posterior (+)/anterior (-) y-axis. The $\mathrm{x}$-axis was parallel to the goal line. The tape was videotaped by all cameras as a calibration object.

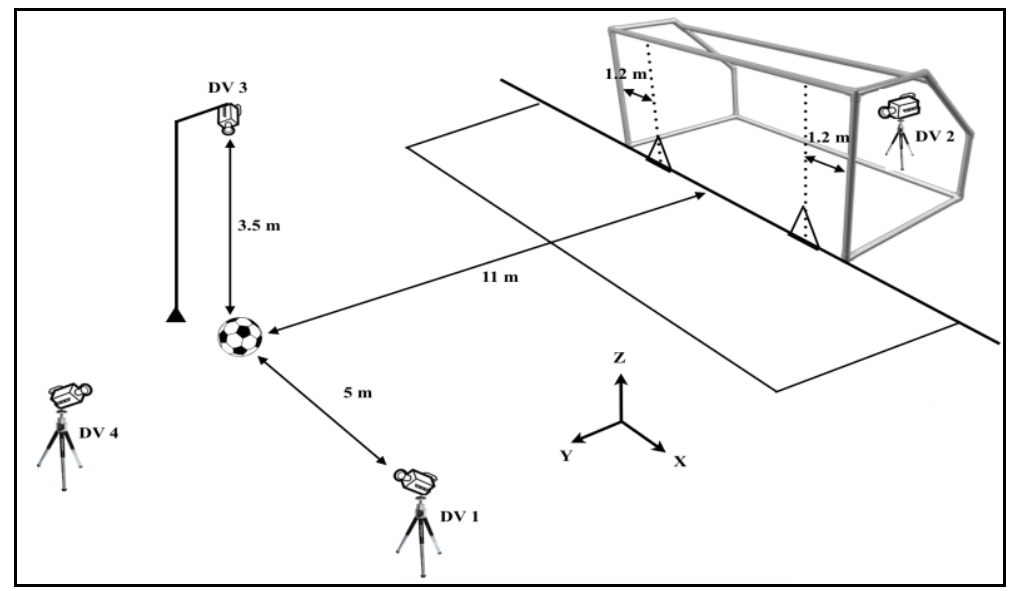

Figure 1. Schematic representation of the data collection set-up. 


\subsection{Testing Procedure}

Prior to the data collection, $15-\mathrm{mm}$ diameter, reflective tapes were placed on participants' anterior superior iliac spine (ASIS) to track the pelvic orientation and improve the digitizing consistency and accuracy. The participants performed about ten minutes of warm-up instructed by their coach including jogging on the field, dribbling the ball and short distance passing. The data were collected on an outdoor soccer field with artificial turf. The goal size was Federation Internationale de Football Association (FIFA) standard: $7.3 \mathrm{~m}$ wide by $2.4 \mathrm{~m}$ high. A FIFA standard sized ball was located at the penalty spot. The participants were instructed to shoot at a target area within the goal using an instep kick as fast and accurately as possible with the right foot. Two target areas (right and left) were defined by the goal post and the vertical axis of a pylon (Figure 1). The pylon was positioned $1.2 \mathrm{~m}$ inside of the left and right goal posts and was 25 $\mathrm{cm}$ high. Participants chose their own preferred approach pattern. Kick direction was alternated every trial and initial direction was counterbalanced across participants. To minimize fatigue, participants took a 20 second break after each trial. The first three trials that place the ball successfully in the target area on each side were selected for further analysis.

\subsection{Data Processing and Analysis}

The Dartfish Team Pro 6.0 (Dartfish, Fribourg, Switzerland) was used to process and analyze the video files. Digitized video clips were from 5 frames before the last step of approach to 5 frames after ball contact. Fourteen points were manually digitized for both sides of the body: the estimated joint center of shoulder, hip, knee and ankle; and toe (tip of the shoe), heel and ASIS. One additional digitized point was the ball center. Test-retest intra-class correlation indicated a high reliability of digitizing process $(\alpha=.95-.98)$. A $12 \mathrm{~Hz}$ fourth-order Butterworth low pass filter was used to filter the raw coordinates data. The cut-off frequency was determined using the residual analysis (Winter, 2009). The kicking phase was defined from kicking foot toe-off to ball contact during the last step of approach. For the sagittal plane, kicking side hip and knee flexion/extension angle and angular velocity were calculated. Hip and knee angles were calculated using relative angles, in which $0^{\circ}$ is the neutral position; flexion is positive and extension is negative. Joint angular velocities were calculated as the first time-derivatives of joint angles. In order to create the ensemble average curve across all participants, the sagittal plane kinematics during the kicking phase were normalized to 21 points period $(0-20)$. Each time interval represented $5 \%$ of the phase, and then all the trials were averaged. For the transverse plane, pelvic orientation (PLO), support foot orientation (SFO), approach angle (APA), distance from support foot heel to ball center in $\mathrm{x}$ and $\mathrm{y}$ direction (DSBx and DSBy) and ball speed were measured/calculated at the instant of the support foot touchdown from the overhead view camera. PLO was measured from the vector between the two ASISs relative to the $\mathrm{x}$-axis (Figure 2). SFO was measured from the vector between toe and heel relative to the y-axis. APA was measured from the orientation of the vector that represented the trajectory of the toe in the transverse plane before touchdown relative to the y-axis. The trajectory of the toe was tracked from three frames before touchdown to the touchdown from the overhead camera. The horizontal ball velocity was calculated as the average speed during the first three frames after ball contact using the finite-difference method (Winter 2009, p.77). The kick direction angle was measured from ball center trajectory to y-axis in the transverse plane after ball contact. For the frontal plane, trunk lateral lean (TKL) and support leg lean (SLL) were measured at the instant of support foot touchdown from the front view camera. TKL was measured from the trunk vector to global vertical axis. The trunk vector was defined as the vector joining the midpoint of the two shoulders and the midpoint of the two ASIS. SLL was measured from the vector between hip and ankle joint to global vertical axis.

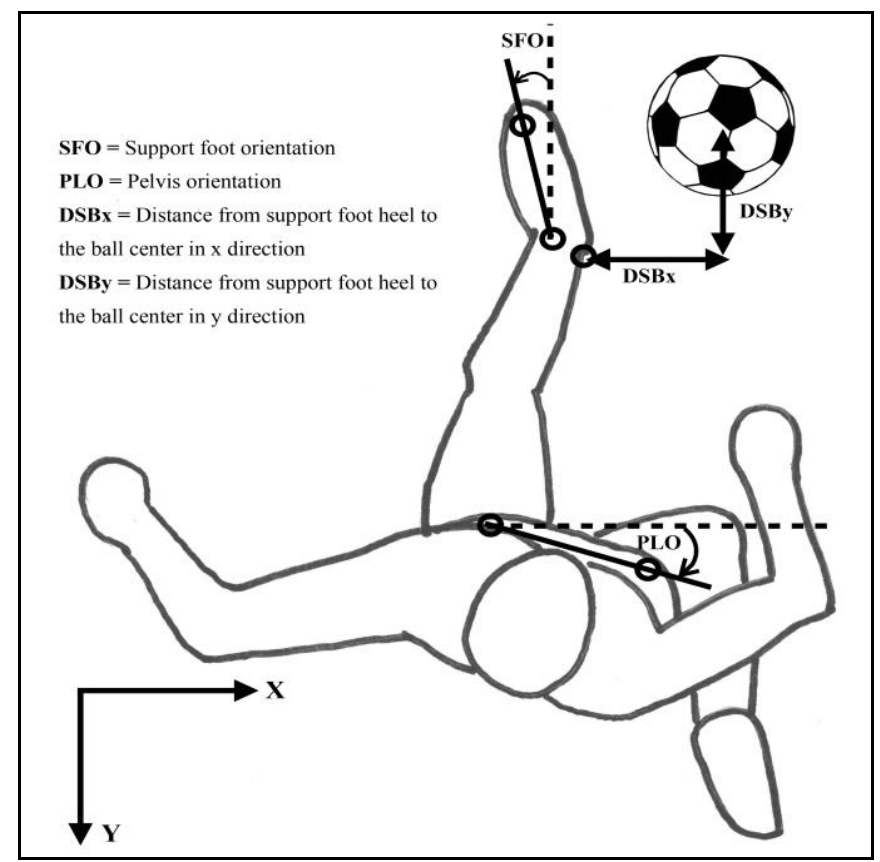

Figure 2. Measurements of kinematic variables at support foot touchdown from the overhead camera. Counterclockwise angle is positive 


\subsection{Statistical Analysis}

Peak values of hip and knee angles and angular velocities, and frontal and transverse plane kinematics at support foot touchdown were tested using paired t-tests between the two kick directions. Stepwise multiple linear regression was used to test whether the kinematic variables predict kick direction angle. Only the variables that were significantly different between the two kick directions were included in the regression analysis. The criterion for statistical significance was $\mathrm{p}<0.05$ for all analyses.

\section{Results}

\subsection{Sagittal Plane Kinematics}

The joint angles and angular velocities for right (kicking side) hip and knee are presented in Figure 3. Support foot touchdown occurred at $43 \%(\mathrm{SD}=3 \%)$ during kicks to the right and at $45 \%(\mathrm{SD}=3 \%)$ during kicks to the left. The peak joint angles and angular velocities in the sagittal plane are presented in Table 1. Paired t-tests indicated that peak knee extension angular velocity was significantly higher in the kicks to the left. No other significant differences were observed.

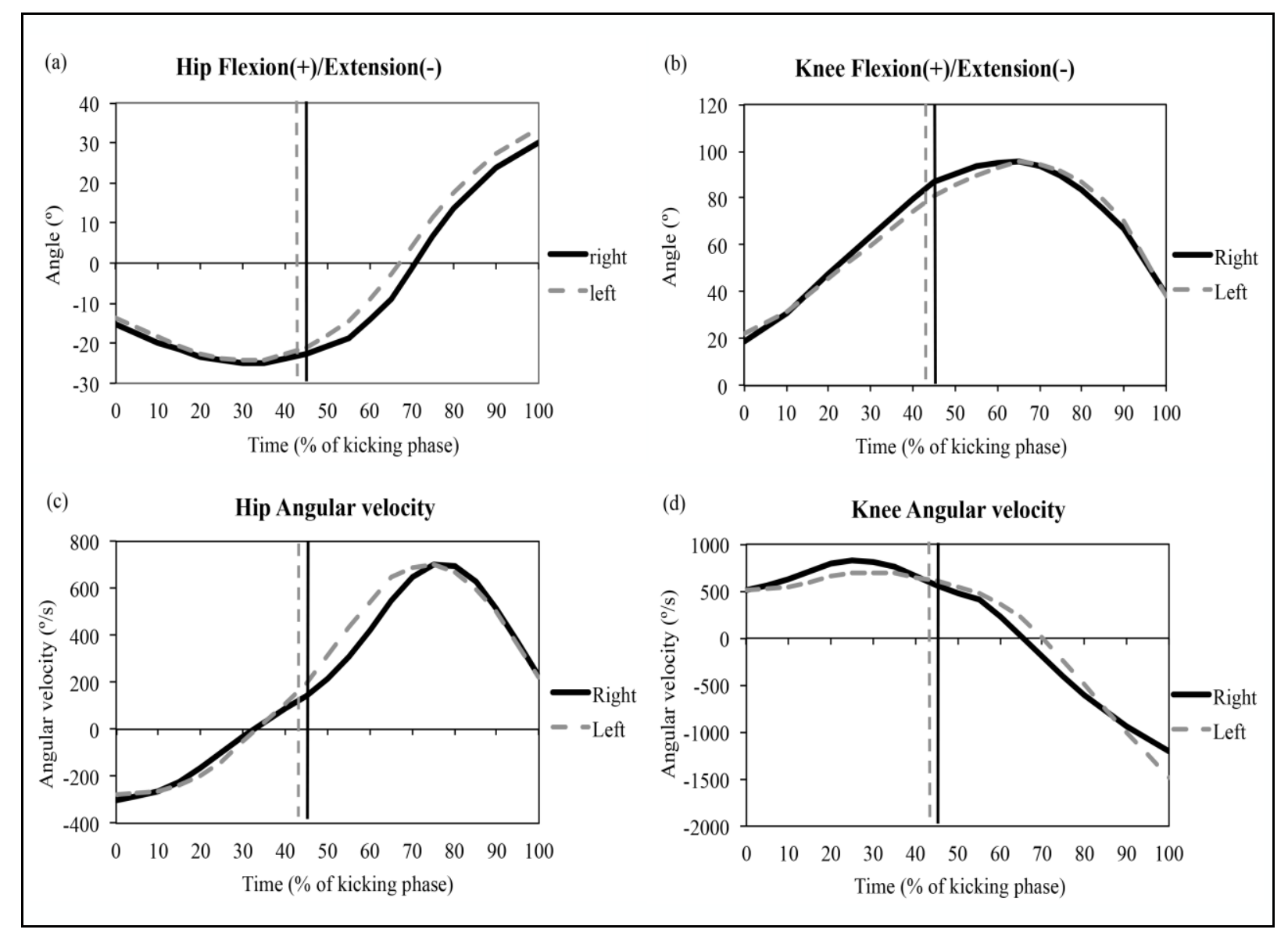

Figure 3. Ensemble average for hip and knee joint angles (a, b) and angular velocities (c, d). $0 \%$ of kicking phase corresponds to toe-off of the right foot (kicking side); $100 \%$ corresponds to ball contact. The support foot touchdown occurred at $43 \%$ and $45 \%$ of kicking phase during the left and right kicks, respectively (shown in vertical lines).

\subsection{Transverse and Frontal Plane}

Kinematic variables in the transverse and frontal plane, and the ball speed and direction measured from the transverse plane are presented in Table 1. The support foot and pelvis pointed more to the left when kicking to the left. In addition, the support heel was significantly closer to (less DSBx) and farther behind (greater DSBy) the ball when kicking to the left. The APA was significantly less for the left kicks. The support leg exhibited significantly less lean for the left kicks. No other statistically significant differences were found. 
Table 1. Lower extremity, pelvis, and ball kinematics (mean \pm SD) displayed in the two kick directions.

\begin{tabular}{|c|c|c|c|c|c|}
\hline & Variables & Right & Left & t-value & p-value \\
\hline \multirow[t]{4}{*}{ Sagittal plane } & Hip extension $\left(^{\circ}\right)$ & $-26 \pm 6$ & $-25 \pm 7$ & 0.354 & 0.731 \\
\hline & Knee flexion $\left(^{\circ}\right)$ & $97 \pm 17$ & $97 \pm 16$ & 0.149 & 0.885 \\
\hline & $\begin{array}{l}\text { Hip flexion } \omega \\
(\%)\end{array}$ & $819 \pm 162$ & $822 \pm 146$ & 0.074 & 0.942 \\
\hline & $\begin{array}{l}\text { Knee extension } \omega \\
(\% / s)\end{array}$ & $-1208 \pm 204$ & $-1490 \pm 321$ & 3.390 & $* 0.007$ \\
\hline \multirow[t]{5}{*}{ Transverse plane } & $\mathrm{SFO}\left({ }^{\circ}\right)$ & $-3 \pm 11$ & $17 \pm 8$ & 12.816 & $*<0.001$ \\
\hline & $\operatorname{PLO}\left({ }^{\circ}\right)$ & $-24 \pm 9$ & $-11 \pm 6$ & 6.746 & $*<0.001$ \\
\hline & $\operatorname{DSBx}(\mathrm{cm})$ & $36 \pm 8$ & $30 \pm 8$ & 6.708 & $*<0.001$ \\
\hline & DSBy $(\mathrm{cm})$ & $5 \pm 10$ & $17 \pm 8$ & 6.591 & $*<0.001$ \\
\hline & $\operatorname{APA}\left({ }^{\circ}\right)$ & $26 \pm 7$ & $8 \pm 6$ & 11.178 & $*<0.001$ \\
\hline \multirow[t]{2}{*}{ Frontal plane } & $\operatorname{TKL}\left({ }^{\circ}\right)$ & $8 \pm 3$ & $7 \pm 2$ & 1.691 & 0.122 \\
\hline & $\operatorname{SLL}\left({ }^{\circ}\right)$ & $28 \pm 8$ & $22 \pm 7$ & 4.279 & $* 0.002$ \\
\hline \multirow[t]{2}{*}{ Ball kinematics } & Ball speed $(\mathrm{m} / \mathrm{s})$ & $17.7 \pm 1.9$ & $17.5 \pm 2.0$ & 1.061 & 0.314 \\
\hline & Kick direction $\left(^{\circ}\right)$ & $-15 \pm 3$ & $14 \pm 3$ & 28.584 & $*<0.001$ \\
\hline
\end{tabular}

For hip and knee joint, flexion is + ; extension is $-. \omega=$ angular velocity. SFO $=$ support foot orientation, PLO = pelvis orientation, DSB = distance from support foot heel to ball center, APA = approach angle, TKL = trunk lateral lean, SLL = support leg lean. The positive sign for SFO indicated the support foot pointed to the left relative to the y axis. The positive sign for the DSBy indicated the heel is behind the ball center. The positive sign for TKL and SLL indicated leaning away from the ball. Right kick direction is negative angle; left is positive. ${ }^{*} p<0.05$.

\subsection{Regression Model}

The kinematic variables that were significantly different between two kick directions included SLL, SFO, PLO, peak knee extension angular velocity, DSBx, DSBy and APA and these were entered to the linear regression model. Through the stepwise procedure, there were three variables that were included in the final regression model: SFO, DSBy and APA. These three variables explained $75.6 \%$ of the variance of kick direction angles $\left(\mathrm{R}^{2}=75.6 \%, \mathrm{p}<.001\right)$. The part correlation squared were $13.7 \%, 6.3 \%$ and $9.0 \%$ for SFP, DSBy and APA, respectively, which indicated how much $\mathrm{R}^{2}$ would decrease if that variable was removed from the regression model (Abdi, 2007). The regression equation produced was as follow:

Kick direction angle $=0.42 \times(S F O)+0.37 \times\left(D S B_{y}\right)-0.62 \times(A P A)+2.47$

\section{Discussion}

\subsection{Support Foot Orientation}

The results of the present study are consistent with reports by previous authors who found that the support foot tends to point toward the desired kick direction (Lees \& Owens, 2011). That is, the SFO positively correlated with the kick direction angle, which indicates that the ball direction would be altered in the same direction as the support foot. The orientation of the support foot may influence the pelvic axial rotation and further affect the kick direction. The proper orientation of the support foot may allow for proper pelvic orientation, which then facilitates a greater range of pelvic rotation needed to achieve maximal velocity of the kicking leg (Lees, Asai, Andersen, Nunome, \& Sterzing, 2010). The pelvis had more retraction angle (greater PLO) and less SFO (more to the right side) in kicks to the right compared to the left. Therefore, SFO could be a useful cue for goalkeepers to predict the kick direction during a penalty kick. SFO appears to be a consistent cue because it barely changed from the support foot touchdown to ball contact (Lees \& Owens, 2011), and may be relatively easy to detect by goalkeepers from the frontal view. The support foot touchdown occurred at about $45 \%$ of the kicking phase, which left about $125 \mathrm{~ms}$ until ball contact. The averaged ball speed in the present study was approximately $17.5 \mathrm{~m} / \mathrm{s}$, which left about $630 \mathrm{~ms}$ from contacting the ball to passing the goal line. With an averaged response time of $200 \mathrm{~ms}$ (from receiving visual cues, e.g. SFO, to initiating the diving movement; Dicks, Button \& Davids, 2010) and an averaged horizontal (medial-lateral) diving speed of $3-4 \mathrm{~m} / \mathrm{s}$ (Suzuki, Togari, Isokawa, Ohashi, \& Ohgushi, 1987), a goalkeeper could cover \pm 1.7 to $\pm 2.2 \mathrm{~m}$ (47\% - 60\% of the goal width) in the medial-lateral direction of the goal. SFO may be beneficial to the goalkeepers who were using the late strategy and give them extra time to anticipate kick direction, but not too early as to be used as deception for penalty takers (Dicks et al., 
2011). However, SFO in the instep kick should be differentiated from those in the curved kick. In a curved kick, the support foot pointed to the right of the desired ball direction (Alcock, Gilleard, Hunter, Baker, \& Brown, 2012).

\subsection{Support Foot Touchdown Position}

The distance from support heel to the ball center at touchdown was found to be different between directions. The support foot touchdown position has been known as a factor that affects the height of the ball trajectory and swing leg orientation (Lees et al., 2010). To our knowledge, there has been no study investigating the role of the support foot touchdown position on kick direction. The linear regression model suggested that the DSBy could significantly predict kick direction. DSBy positively correlated with kick direction angle, which indicates that the more backward the support heel touchdown the more toward the left side the ball would go. Even though the effect of touchdown position on the ball direction may be subtle, along with the SFO, it may provide information to predict the kick direction. It is likely that if the support foot touchdown is behind the ball and points towards the left side, the ball will probably go to the left. If the support foot touchdown is in front of the ball and points towards the right, the ball will probably go to the right. Therefore, we suggested that future penalty defense training for goalkeepers may focus on searching visual information from penalty taker's support foot touchdown. Our suggestion was supported by Savelsbergh et al. (2002) who used an eye-tracking system and observed that the expert goalkeepers spent longer fixating on the kicking leg, support leg and ball areas.

\subsection{Approach Angle}

A $30-45^{\circ}$ approach relative to the desired ball direction produces maximal ball speed (Isokawa \& Lees, 1988). An angled approach can position the body to gain greater hip and knee flexion and enables the kicking leg to be tilted in the frontal plane so that the foot can be placed further under the ball, thus enabling better ball contact (Lees \& Nolan, 1998). The players altered APA depending on kick direction. In the present study, the APA was significantly greater in the kicks to the right side, meaning that APA is a potentially valuable source of information for goalkeepers. The regression model indicated that the APA negatively correlated with kick direction, which means that the greater APA the more to the right side the ball would go. Though APA is a significant predictor, however, there may be difficulties to use APA as information to predict the kick direction. Firstly, the desired APA varies from player to player. A previous study reported self-selected APA was $30.3 \pm 15.2^{\circ}$ with range of $39^{\circ}$ during instep kick (Scurr \& Hall, 2009). Secondly, the approach angle may be difficult to detect from the front view or judge as large or small by goalkeepers due to lack of consistent reference. Thirdly, Dicks et al. (2011) argued that the APA was an early piece of information that can be used as deception for penalty kickers to fool the goalkeepers. Therefore, APA may be a less consistent or reliable predictor compared to SFO and DSBy for anticipating kick direction. When APA was removed from the regression model, the explained variance would drop to about $67 \%$ (dropped by $9 \%$ from $75.6 \%$ ).

Other variables (e.g. PLO, TKL, SLL and peak knee extension angular velocity) could likely account for the unexplained variance of kick direction (about 24\%). However, these variables are difficult to detect or judge by goalkeepers during such a fast kick movement; thus, less valuable for anticipating kick direction.

\subsection{Limitations}

One limitation of the present study is the conventional two-dimensional (2D) analysis that was used in the study. Nunome \& Ikegami (2006) observed a distortion in knee angular velocity $50 \mathrm{~ms}$ before ball contact by the 2D analysis compared with 3D. They suggested this distortion was likely caused by computing angular velocities from quasi-planar projection (Nunome \& Ikegami, 2006). However, it is unclear that such distortion displayed in the present study. Another potential limitation is the relatively low number of participants and trials for each participant, which may increase the type II error. Even with the low sample size, however, several significant differences were observed. A final limitation is that the present study did not examine the kinematics of kicks to the center of the goal. It has been reported that $28.7 \%$ of kick directions in skilled players were to the center (Bar-eli et al., 2007). For future studies, analyzing more directions would provide more insight to role of kinematics in predicting kick direction.

\section{Conclusion and Practical Applications}

In this study, differences in kinematics were observed between penalty kick directions. Support foot orientation along with support foot placement behind the ball may be the most useful cues to anticipate kick direction for goalkeepers in penalty defense. The support foot tended to point towards the desired kick direction and planted further behind the ball in the left kicks. Other factors may be less informative to be used as visual cues.

\section{References}

Abdi, H. (2007). Part (semi partial) and partial regression coefficients. In Neil Salkind (Ed.), Encyclopedia of Measurement and Statistics (735-737). Thousand Oaks (CA): Sage.

Alcock, A. M., Gilleard, W., Hunter, A. B., Baker, J., \& Brown, N. (2012). Curve and instep kick kinematics in elite female footballers. Journal of Sports Sciences, 30(4), 387-394. http://dx.doi.org/10.1080/02640414.2011.643238

Andersen, T. B., \& Dörge, H. C. (2011). The influence of speed of approach and accuracy constraint on the maximal speed of the ball in soccer kicking. Scandinavian Journal of Medicine \& Science in Sports, 21(1), 79-84. http://dx.doi.org/10.1111/j.1600-0838.2009.01024.x 
Bar-eli, M., Azar, O. H., \& Ritov, I. (2007). Action bias among elite soccer goalkeepers : The case of penalty kicks. Journal of Economic Psychology, 28, 606-621. http://dx.doi.org/10.1016/j.joep.2006.12.001

Dicks, M., Button, C., \& Davids, K. (2010). Effects of availability of advance visual information on association football goalkeeping performance during penalty kicks. Perception, 39(8), 1111-1124.

Dicks, M., Uehara, L., \& Lima, C. (2011). Deception, Individual Differences and Penalty Kicks: Implications for Goalkeeping in Association Football. International Journal of Sports Science and Coaching, 6(4), 515-522. http://dx.doi.org/10.1260/1747-9541.6.4.515

Dörge, H. C., Andersen, T. B., Sørensen, H., \& Simonsen, E. B. (2002). Biomechanical differences in soccer kicking with the preferred and the non-preferred leg. Journal of Sports Sciences, 20(4), 293-299.

Isokawa, M., \& Lees, A. (1988). A biomechanical analysis of the instep kick motion in soccer. Science and Football: Proceedings of the First World Congress of Science and Football, 449-455.

Katis, A., \& Kellis, E. (2010). Three-dimensional kinematics and ground reaction forces during the instep and outstep soccer kicks in pubertal players. Journal of Sports Sciences, 28(11), 1233-1241. http://dx.doi.org/10.1080/02640414.2010.504781

Kuhn, W. (1987). Penalty-kick strategies for shooters and goalkeepers. In Science and football: proceedings of the First World Congress of Science and Football, Liverpool, UK (pp. 489-492).

Lees, A., \& Nolan, L. (1998). The biomechanics of soccer: a review. Journal of Sports Sciences, 16(3), 211-234. http://dx.doi.org/10.1080/026404198366740

Lees, A., Asai, T., Andersen, T. B., Nunome, H., \& Sterzing, T. (2010). The biomechanics of kicking in soccer: a review. Journal of Sports Sciences, 28(8), 805-817. http://dx.doi.org/10.1080/02640414.2010.481305

Lees, A., \& Owens, L. (2011). Early visual cues associated with a directional place kick in soccer in soccer. Sports Biomechanics, 10(02), 125-134. http://dx.doi.org/10.1080/14763141.2011.569565

Morya, E., Bigatao, H., Lees, A., \& Ranvaud, R. (2005). Evolving penalty kick strategies: World Cup and club matches, 2000-2002. In Science and football V. Proceedings of the fifth world congress of science and football (p. 510).

Nunome, H., Asai, T., Ikegami, Y., \& Sakurai, S. (2002). Three-dimensional kinetic analysis of side-foot and instep soccer kicks. Medicine and Science in Sports and Exercise, 34(12), 2028-2036. http://dx.doi.org/10.1249/01.MSS.0000039076.43492.EF

Nunome, H., \& Ikegami, Y. (2006). Kinematics of soccer instep kicking: A comparison of two-dimensional and threedimensional analysis. Proceedings Of International Symposium On Biomechanics In Sports, 2611-2614.

Savelsbergh, G., Williams, A., Van Der Kamp, J., \& Ward, P. (2002). Visual search , anticipation and expertise in soccer goalkeepers Visual search, anticipation and expertise in soccer goalkeepers. Journal of Sports Sciences, (20), 279-287.

Scurr, J., \& Hall, B. (2009). The effects of approach angle on penalty kicking accuracy and kick kinematics with recreational soccer players. Journal of Sports Science \& Medicine, 8(2), 230-234.

Suzuki, S., Togari, H., Isokawa, M., Ohashi, J., \& Ohgushi, T. (1987). Analysis of the goalkeeper's diving motion. In T. Reilly, A. Lees, K. Davids, \& W. J. Murphy (Eds.) Science and Football. Proceedings of the $1^{\text {st }}$ World Congress of Science and Football, Liverpool, UK, 13-17 April (pp. 468-475). Routledge Revivals.

Van der Kamp, J. (2006). A field simulation study of the effectiveness of penalty kick strategies in soccer: late alterations of kick direction increase errors and reduce accuracy. Journal of Sports Sciences, 24(5), 467-477. http://dx.doi.org/10.1080/02640410500190841

Winter, D. A. (2009). Biomechanics and motor control of human movement. Hoboken, N.J. : Wiley, c2009. 